\title{
Comparing the Effectiveness of TAT and Myristoylation of gp91ds on Leukocyte Superoxide (SO) Release
}

\author{
Harsh Patel, Kyle Bartol, Amelie Bottex, Ryan Remarcke, William Chau, \\ Sydney Walker, Qian Chen, Robert Barsotti, and Lindon Young \\ Department of Bio-Medical Sciences, Philadelphia College of Osteopathic Medicine, \\ 4170 City Avenue, Philadelphia, PA, 19131, USA
}

\section{Introduction}

SO release from leukocytes via NADPH oxidase activation contributes to oxidative stress under various diseases, such as ischemia/reperfusion (I/R) injury and vascular complications in diabetes. NADPH oxidase has seven isoforms with NOX2 being the predominant isoform of NADPH oxidase in polymorphonuclear leukocytes (PMNs). Activation of NOX2 requires the assembly of cytosolic subunits (p47 $\left.7^{\text {phox }}, \mathrm{p} 40^{\text {phox }}, \mathrm{p} 67^{\text {phox }}, \mathrm{Rac}\right)$ to plasma membrane subunits (gp91 ${ }^{\text {phox }}$ and $\mathrm{p} 22^{\text {phox }}$ ) [1]. NADPH oxidase is activated during $\mathrm{I} / \mathrm{R}$ injury via cytokine receptor stimulation or chemotactic factor (N-formyl-L-methionyl-L-leucyl-L-phenylalanine (fMLP, MW= $438 \mathrm{~g} / \mathrm{mol}$ ) and utilizes molecular oxygen to produce SO [2] (Figure 1).

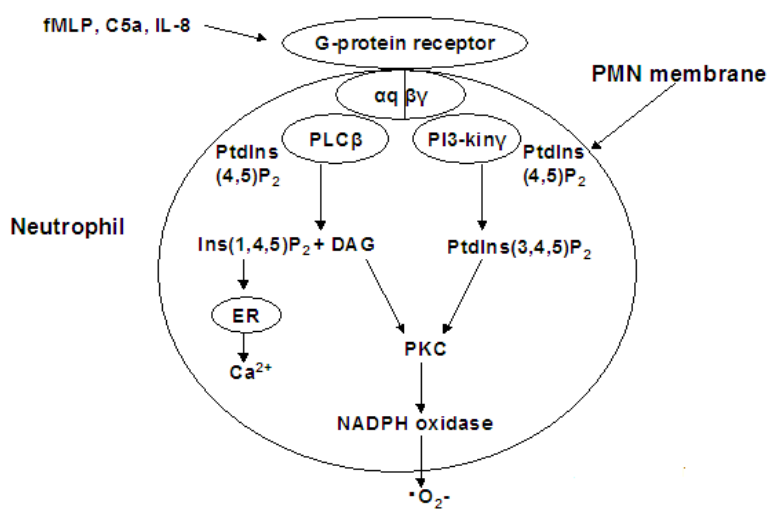

Fig. 1. Schematic representation of PKC activation generating $S O$ release in PMN. $P M N$ chemotactic G-protein receptors are activated by fMLP, C5a and interleukin 8. The G-protein subunits $\alpha q$ and $\beta \gamma$ disassociate after stimulation and activate phospholipase $C$ beta (PLC $\beta$ ) and phosphatidyl inositol-3-kinase gamma (PI-3kin $\gamma)$ to produce inositol 1,4,5 trisphosphate $\left(\operatorname{Ins}(1,4,5) P_{2}\right)$ plus DAG and PtdIns $(3,4,5) \mathrm{P}_{2}$ respectively from phospholipids phosphatidylinositol 4,5 bisphosphate (PtdIns $\left.(4,5) P_{2}\right)$. Ins $(1,4,5) P_{2}$ stimulates $\mathrm{Ca}^{2+}$ release from the endoplasmic reticulum (ER). $\mathrm{Ca}^{2+} / D A G$

and PtdIns $(3,4,5) P_{2}$ directly activate PKC. Activated PKC phosphorylates NADPH oxidase to release $\mathrm{SO}$ anion $\left(\bullet \mathrm{O}_{2}-\right)$. Adapted from Young, et al. [2].

Gp91ds conjugated with tat or myristric acid + pegylated linker (myr-peg) are cell permeable peptide formulations which selectivity inhibit NADPH oxidase assembly by blocking $\mathrm{p} 47^{\text {phox }}$ interaction with gp91 $1^{\text {phox }}$ (Figure 2).

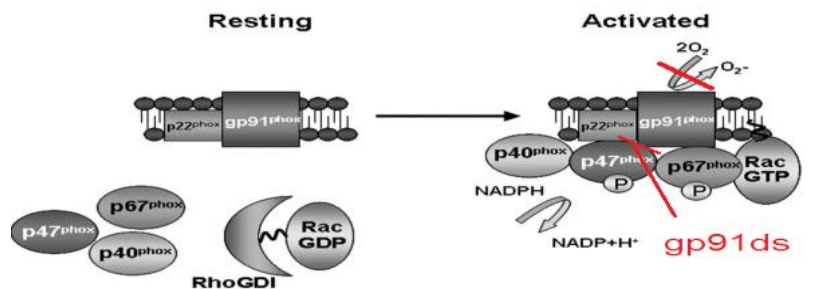

Fig. 2. Schematic showing the inactive and active forms of NADPH oxidase. Red-lines denote areas of inhibition performed by gp91ds. Adapted from Wilkinson, et al. [1]

It is well known that adding myristic acid or a tat carrier peptide to native peptides facilitates cell membrane permeability which is required for effectively targeting intracellular substrates. The 
addition of a glycine-glycine ( $\mathrm{gg}$ ) spacer between the tat and cargo portion of the peptide is reported to facilitate delivery of the cargo sequence (i.e., CSTRIRRQL) [3,4] (Figure 3).

A

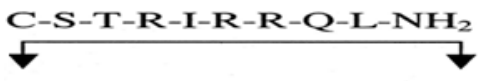

gP91ds portion

$\mathrm{C}$

[H]-R-K-K-R-R-Q-R-R-R-C-S-T-R-I-R-R-Q-L-NH

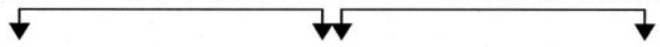

tat portion

gp91ds portion

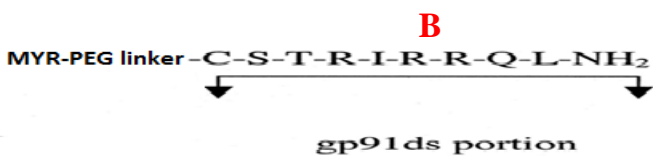

D

[H]-R-K-K-R-R-Q-R-R-R- G-G-C-S-T-R-I-R-R-Q-L-NH

tat portion

gp91ds portion

Fig. 3. A: native gp91ds $(M W=1131 \mathrm{~g} / \mathrm{mol})$. B: myr-peg linker-gp91ds $(\mathrm{MW}=1486 \mathrm{~g} / \mathrm{mol})$. C: $\mathrm{gp} 91 \mathrm{ds}$ tat sequence $(M W=2452 \mathrm{~g} / \mathrm{mol})$. D: gp9lds-tat gg spacer $(M W=2566 \mathrm{~g} / \mathrm{mol})$. Adapted from Rey, et al. [3].

We have previously shown that myristic acid conjugated caveolin-1 and protein kinase C (PKC) beta II and zeta peptide inhibitors significantly attenuated fMLP-induced SO release compared to their native counterparts [5]. However, it is not known if differences exist in the effectiveness of myristic acid versus tat conjugated gp91 ds-tat peptides compared to their native counterparts or untreated controls. We hypothesized that myr-gp91ds $(2-10 \mu \mathrm{M})$ would dose-dependently attenuate fMLP induced PMN SO release at lower concentrations compared to tat conjugated or tat conjugated gg spacer-gp91ds peptides. Moreover, we also predict that both myristic acid and tat conjugated gg spacer gp91ds peptides would significantly attenuate fMLP-induced leukocyte SO release compared to native or untreated controls without affecting cell viability.

\section{Results and Discussion}

\section{Isolation of PMNs}

Male Sprague-Dawley rats (350-400 g, Charles River), used as PMN donors, were anesthetized with $2.5 \%$ isoflurane and given a $16 \mathrm{ml}$ intraperitoneal injection of $0.5 \%$ glycogen (Sigma Chemical) dissolved in PBS. Approximately $16 \mathrm{~h}$ later, the rats were reanesthetized with isoflurane and the PMNs were harvested by peritoneal lavage in $30 \mathrm{ml}$ of $0.9 \% \mathrm{NaCl}$, as previously described [2,5].

\section{Measurement of SO Release from Rat PMNs}

The SO release from PMNs was measured spectrophotometrically (Gilford model 260, Nova Biotech; El Cajon, CA) by the reduction of ferricytochrome c [2,5]. The PMNs $\left(5 \times 10^{6}\right.$ cells $)$ were suspended in $450 \mu \mathrm{l}$ PBS and incubated with $100 \mu \mathrm{M}$ ferricytochrome c (Sigma Chemical; St Louis, MO) in a total volume of $900 \mu \mathrm{l}$ PBS in the presence or absence of myr-peg conjugated (2 to $10 \mu \mathrm{M}$ ), tat conjugated $(80 \mu \mathrm{M})$ or native gp91ds $(80 \mu \mathrm{M})$ for $15 \mathrm{~min}$ at $37^{\circ} \mathrm{C}$ in spectrophotometric cells. Thereafter, absorbance at $550 \mathrm{~nm}$ was measured every $30 \mathrm{sec}$ for up to $120 \mathrm{sec}$ following fMLP $1 \mu \mathrm{M}$ (Calbiochem; La Jolla, CA) stimulation and the change in absorbance (SO release from PMNs) was determined relative to time 0 in a final reaction volume of $1 \mathrm{ml}$. Cell viability among all study groups was determined by $0.3 \%$ trypan blue exclusion at the end of the SO release assay. 
Myr-peg gp91 $(5 \& 10 \mu \mathrm{M})$ significantly attenuated untreated and native gp91ds induced SO release by $56-57 \pm 8 \%$ and $52-54 \pm 9 \%$ respectively. Myr-peg gp91 $(2 \mu \mathrm{M})$, gp91ds-tat $(80 \mu \mathrm{M})$, and gg spacer $(80 \mu \mathrm{M})$ significantly inhibited untreated by $36 \pm 11 \%$ compared to untreated controls and was not different from native gp91ds (see Figure 4). Concentrations of tat conjugated peptides $\leq 40 \mu \mathrm{M}$ did not exert significant inhibition of PMN SO release. (Data not shown)

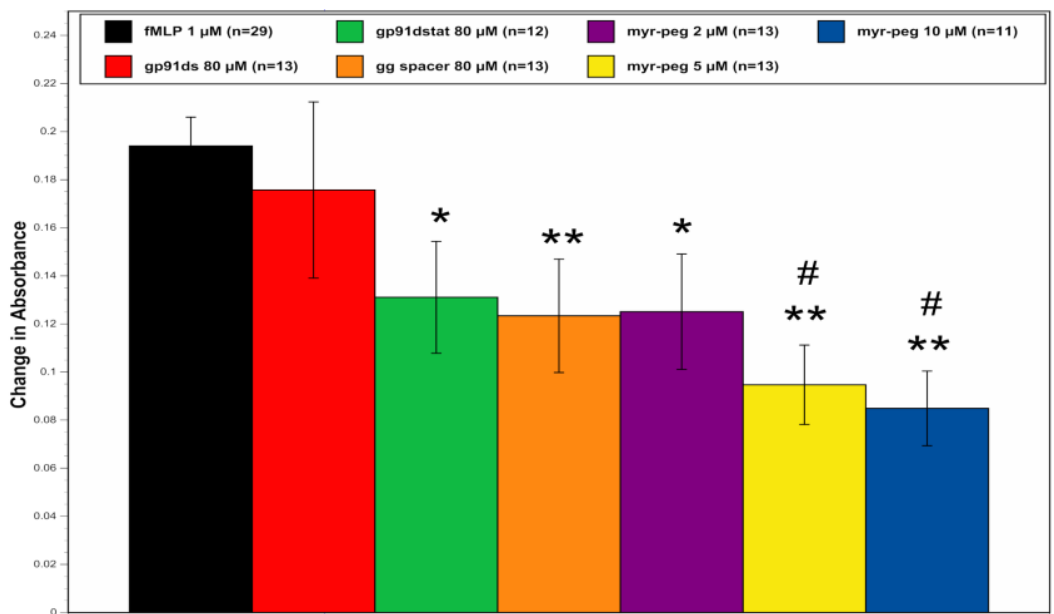

Fig. 4. fMLP (1 $\mu M)$-induced peak response SO release in PMNs. Probability values of $<0.05$ are considered to be statistically significant $\left({ }^{*} p<0.05, * * p<0.01\right.$ compared to untreated controls; ${ }^{*} p<0.05$ compared to native gp91ds). All data in the text and figures are presented as means \pm S.E.M. The data were analyzed by analysis of variance using post hoc analysis with the Fisher's test and n= number of trials.

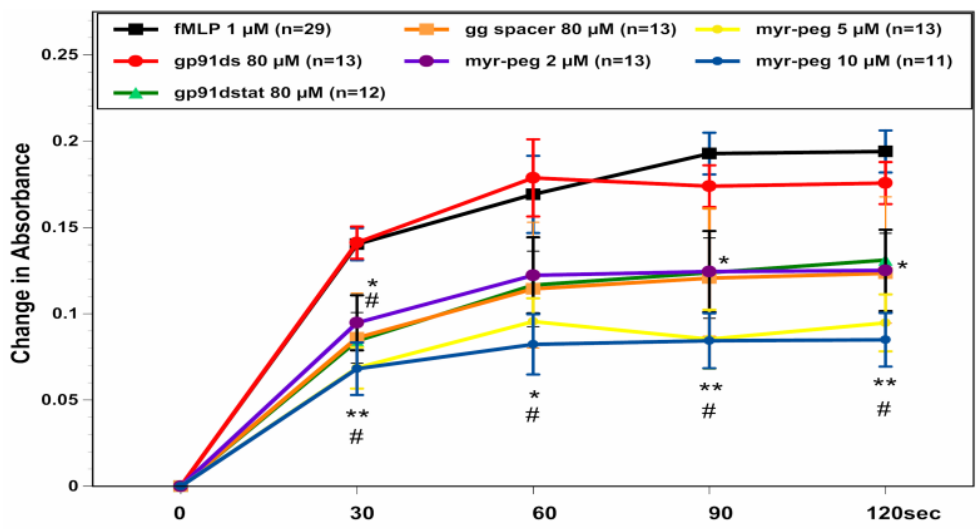

Fig. 5. The time course response of $f M L P(1 \mu M)$-induced SO release. ${ }^{*} p<0.05$, $* * p<0.01$ compared to untreated controls; ${ }^{\#} p<0.05$ compared to native gp $91 \mathrm{ds}$.

Myr-peg gp91 (5 \& $10 \mu \mathrm{M})$ significantly inhibited untreated and native gp91 by $44-56 \%$ and $46-52 \%$ respectively from 30-120 sec. Myr-peg gp91ds $(2 \mu \mathrm{M})$, gp91dstat $(80 \mu \mathrm{M})$, and gp91ds-tat with gg spacer $(80 \mu \mathrm{M})$ significantly untreated by $33-36 \%$ from $30-120 \mathrm{sec}$ and was only different from native gp91 at $30 \mathrm{sec}$ (Figure 5).

We also observed that TAT (R-K-K-R-R-Q-R-R-R; MW=1321g/mol, $\mathrm{n}=7$ ) alone decreased fMLP-induced absorbance by $48 \pm 9 \%$ and cell viability was $92 \pm 1 \%$ (data not shown). Although cell viability was not significantly different from untreated and other tat conjugated gp91ds groups, we observed a significant degree of cell clumping, which in turn would decrease the absorbance. Therefore 
the measured decrease in absorbance was likely due to the effect of cell clumping rather than a decrease in fMLP induced SO release. By contrast, cell clumping was not observed in the other tat conjugated gp91ds groups suggesting that in these groups leukocyte SO release was attenuated. These results suggest that tat alone may potentiate cell aggregation.

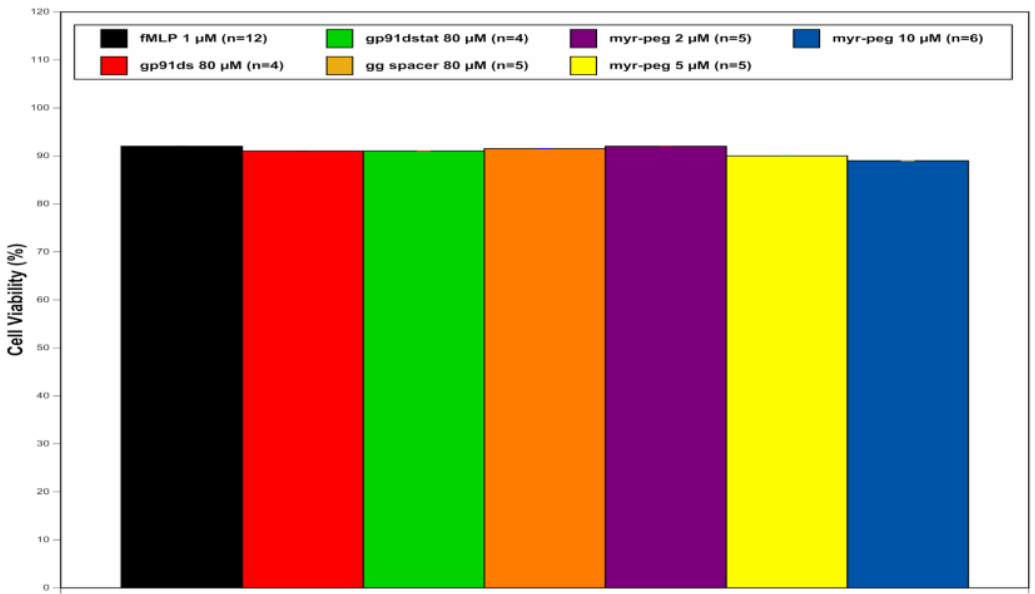

Fig. 6. The effects of native, tat, and myr-peg conjugated gp91ds on cell viability in $f M L P(1 \mu M)$ induced $S O$ release in PMNs. Cell viability

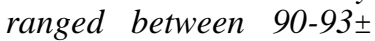
$1 \%$ and was not different amongst study groups indicating that the reduction in $S O$ release was not related to cell death.

Unconjugated native sequence did not inhibit the fMLP induced SO response at the highest dose tested $(80 \mu \mathrm{M})$. Myr-peg gp91ds NADPH oxidase peptide inhibitor significantly attenuated leukocyte SO release dose dependently compared to untreated or native sequence (myr-peg-gp91ds; $2-10 \mu \mathrm{M})$. The tat conjugated gp91ds inhibitors (both $80 \mu \mathrm{M}$ ) significantly attenuated fMLP-induced leukocyte SO release, but to a lesser extent than the myr-peg linked inhibitor and were not different from native gp91ds at 60-120 sec. Moreover, we expected to see greater inhibition with gp91ds-tat with gg spacer compared to gp91ds-tat since the gg spacer is reported to facilitate delivery of the cargo sequence. However, this subtle difference in cargo sequence delivery may be masked with respect to inhibition of leukocyte SO release in this assay. Collectively, these results suggest that native gp91ds requires conjugation of myr-peg or tat in order to effectively inhibit the intracellular target of p47 ${ }^{\text {phox }}$ and gp91 interaction. Additionally, myr-peg-gp91ds is more cell permeable and therefore can inhibit fMLPinduced SO release from leukocytes at lower doses compared to gp91ds-tat and gg spacer gp91ds-tat. Future studies will be aimed at comparing myr and tat conjugated peptides that have other intracellular targets i.e. mitochondria in myocardial ischemia-reperfusion.

\section{Acknowledgments}

This study was supported by the Center for Chronic Disorders of Aging and the Department of Bio-Medical Sciences at PCOM. Special thanks to PCOM DO/Biomed students Kevin Kucharski, Stephanie Liu and Gregory Stoner for assistance with editing the manuscript.

\section{References}

1. Wilkinson, B.L., Landreth, G.E. J. Neuroinflammation 3, 30 (2006), http://dx.doi.org/10.1186/1742-2094-3-30

2. Young, L.H., et al. Cardiovascular Drug Reviews 23, 255-272 (2005),

http://www.ncbi.nlm.nih.gov/pubmed/16252018

3. Rey, F.E., et al. Circulation Research 89, 408-414 (2001), http://www.ncbi.nlm.nih.gov/pubmed/?term=Novel+Competitive

4. Taraballi, F., et al. Frontiers in Neuroengineering (2010), http://dx.doi.org/10.3389/neuro.16.001.2010

5. Perkins, K.A., et al., in Lebl, M. (Ed.) Peptides: Building Bridges (Proceedings of the 22nd American Peptide Symposium), American Peptide Society, San Diego, 2011, p. 288-289, http://www.5z.com/22APS/proceedings/22APS_full_color_v5.pdf 